\title{
Impact of the manufacturing technique on the dissolution-enhancement functionality of PEG4000 in Cilostazol tablets
}

\author{
Obada Sawafta ${ }^{1}$, Sonia Alhadid ${ }^{1}$, Izz Aldeen Abu Awwad², Eman Migdadi ${ }^{1}$, Ahmad Aljaberi ${ }^{1}$ \\ 1 Department of Pharmaceutical Sciences and Pharmaceutics, Faculty of Pharmacy, Applied Science Private University, Amman, Jordan \\ 2 Tabuk Pharmaceutical Research Company, Amman, Jordan \\ Corresponding author: Ahmad Aljaberi (aljaberi@asu.edu.jo)
}

Received 25 December 2020 • Accepted 1 February 2021 • Published 22 February 2021

Citation: Sawafta O, Alhadid S, Abu awwad IA, Migdadi E, Aljaberi A (2021) Impact of the manufacturing technique on the dissolution-enhancement functionality of PEG4000 in Cilostazol tablets. Pharmacia 68(1): 243-250. https://doi.org/10.3897/ pharmacia.68.e62465

\begin{abstract}
Cilostazol was selected as poorly-soluble model drug to investigate the impact of the manufacturing method on the excipient functionality of PEG4000 at various levels. Powder blends were prepared by direct compression (DC), wet granulation (WG) and hot-melt extrusion (HME). Characteristics of these blends and their compressed tablets were investigated by standard techniques. Solid-state characterization was carried out using differential scanning calorimetry (DSC). While DC trials were found with no significant differences, WG and HME showed contrasting enhancement and retardation effects regarding the dissolution profile of Cilostazol tablets depending on the level of PEG4000 incorporated. The optimal enhancement of dissolution was obtained at 10\% w/w PEG4000 for tablets prepared by HME. DSC analysis indicated that no solid solutions were formed at such low levels of PEG4000during processing by either manufacturing techniques. Consequently, the wetting functionality and dissolution enhancement of PEG4000 was revealed to be level- and manufacturing method dependent.
\end{abstract}

\section{Keywords}

Hot-melt extrusion, Cilostazol, PEG4000, Dissolution; excipient functionality

\section{Introduction}

APIs with poor solubility represent $40 \%$ of the marketed drug entities and almost $90 \%$ of molecules in the pipeline of discovery (Repka et al. 2008; Kalepu and Nekkanti 2015). For many drug substances, conventional methods of formulating tablets dosage forms such as direct compression and wet granulation provide clinically effective therapy while keeping the required balance of pharmacodynamic and pharmacokinetic profiles without any effect on patient's safety. However, there are some limitations to the use the conventional methods of formulation with poorly soluble drugs. Moreover, few APIs imply the necessary characteristics during the tableting process. To overcome the poor solubility as well as the poor physical characteristics of some APIs, tablets manufacturing could imply complex processes or rather different manufacturing approaches.

Hot-melt extrusion (HME), is an example of such techniques that were proven as a viable technique in pharmaceutical manufacturing. HME technique has been demonstrated to be applicable in preparing immediate release granules, sustained-release tablets, transdermal and transmucosal drug delivery systems (Regev et al. 2019; Ren et 
al. 2019; Yang et al. 2019; Tang et al. 2019; Sanoufi et al. 2020). Several pharmaceutical products manufactured by HME technique are now commercially available such as Nurofen, Isoptin SR-E and Kaletra (Maniruzzaman et al. 2012). The vast majority of HME studies for solubility enhancement were trying to solve the poor solubility problem by changing the molecular level of the API and forming solid dispersions by incorporating hydrophilic carriers (Leuner and Dressman 2000; Albers et al. 2009; Kalivoda et al. 2012; Weatherley et al. 2013; Zi et al. 2019). Different types of dispersions can be obtained using melt extrusion of a drug within a polymer in a binary mixture. i.e. the drug could be dispersed molecularly within the carrier (polymer) or exists on its amorphous or crystalline original phase. In such an approach, a high polymer to drug ratio is usually required to maintain the physical stability of the solid extrudates whether the drug is solubilized or dispersed (Patil et al. 2016).

However, the usefulness of HME technique in enhancing drug dissolution simply through improved wettability and solubilization upon using hydrophilic polymers at a low polymer to drug ratio was overlooked. In addition, few studies have put HME into comparison with other conventional methods regarding their effect on the solubility and dissolution for formulations incorporating hydrophilic polymers. Therefore, the aim of this work was to study and clarify the effect of PEG4000 as a hydrophilic polymer on the dissolution of Cilostazol as a model poorly soluble drug in a tablet dosage form (Figure 1). Additionally, this work aimed to clarify how the different manufacturing technologies may contribute to Cilostazol dissolution without changing the crystalline form of the API.

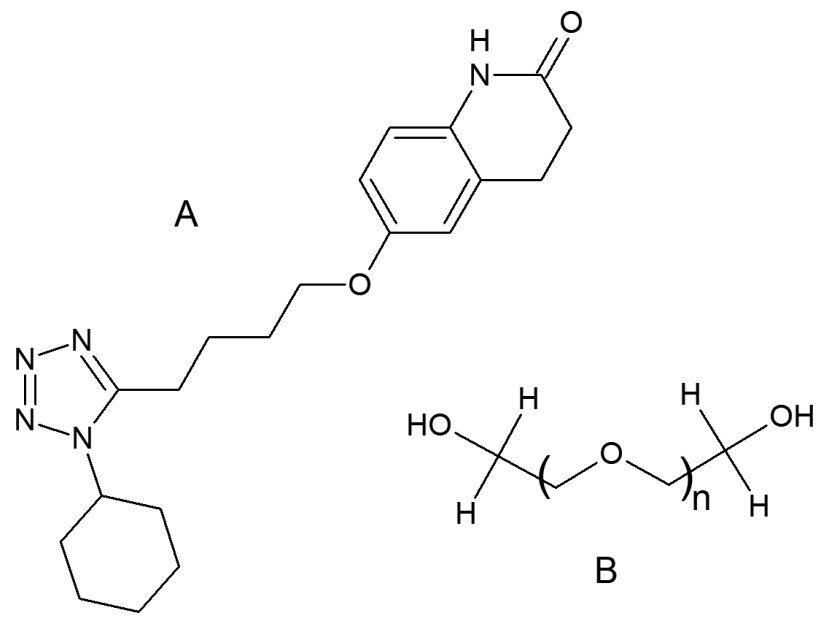

Figure 1. The chemical structure of (A) Cilostazol and (B) PEG4000. The average value of $n$ in PEG4000 is 90 .

\section{Methodology}

\section{Abbreviations}

DC, Direct compression; DSC, Differential scanning calorimetry; API, Active Pharmaceutical Ingredients; CI, Carr's index; GRAS, Generally regarded as safe; HME,
Hot-melt extrusion; HPMC, Hydroxy-propyl-methyl-cellulose; PSD, Particle Size Distribution; PEG, Polyethylene Glycol; RSD, Relative Standard Deviation; SD, Standard Deviation; WG, Wet Granulation; LOD, loss on drying.

\section{Materials}

Cilostazol used in the study was produced by Cadila LTD Company (Ahmedabad, India), PEG 4000 was from by Seppic company (La Garenne-Colombes, France). Avicel PH102 was from FMC Corporation (Philadelphia, PA, USA). Lactose Monohydrate was from DFE Pharma Company (Tamil Nadu, India). Carmellose Calcium was from Nichirin Company (Hyōgo Prefecture, Japan). Colloidal Silicon Dioxide was from Evonik (Essen, Germany). Magnesium Stearate was from Merck (New Jersey, USA). Sodium lauryl sulfate was from Sigma Aldrich (St. Louis, MO, USA).

\section{Direct compression process}

A standardized formula of Cilostazol tablets was prepared by DC technique using the composition of the prototype formula, named DC01 (Table 1). The standard formula was performed without the incorporation of PEG4000. Briefly, Cilostazol was mixed with Lactose. The mixed blend was sieved through \# 30 mesh sieve. Avicel PH102, colloidal silicon dioxide and carmellose calcium were de-lumped prior to addition by pre-sieving through \# 30 mesh sieves. All of the components of each formulation were accurately weighed, mixed in a polyethylene bag for 10 minutes manually to obtain a uniform dry blend. Magnesium Stearate at $1 \% \mathrm{w} / \mathrm{w}$ level was added to the blend prepared above and the components were mixed for an additional $5 \mathrm{minu}-$ tes. All other trials were accomplished in the same way but with addition of the hydrophilic polymers with the quantity specified in each formulation. PEG4000 flakes were milled using an automatic grinder (Sanyo blinder, Sanyo Electrics Co. LTD, Japan) prior to use. The tableting process involved a rotary compression machine (Cad-mackCambert Compression machine) equipped with rounded $9.0 \mathrm{~mm}$ in diameter punches and their die.

\section{Wet granulation process}

A standardized formula of Cilostazol tablets was prepared by WG technique using the composition of the prototype formulation mentioned previously. This trial was named WG01. Cilostazol, Lactose Monohydrate, and PEG4000 were sieved through \# 30 mesh and mixed for 10 minutes. The blend was granulated manually with Purified water until satisfactory massed granules were produced. The granules were dried in an oven at $40{ }^{\circ} \mathrm{C}$ to a loss on drying (LOD) value less than $3.0 \%$ in order to get strong granules that aid the manufacturing process. The granules were milled using Sanyo grinder and then passed through \# 30 mesh. Extra-granular components were added as described previously for the DC trials. Tablets were compressed 
Table 1. The composition for Cilostazol tablets prepared by various manufacturing techniques.

\begin{tabular}{|c|c|c|c|c|c|c|c|c|}
\hline & DC01 & DC02 & DC03/HME02 & DC04/HME03 & WG01 & WG02 & WG03 & WG04 \\
\hline Material name & $\mathrm{w} / \mathrm{w} \%$ & $\mathrm{w} / \mathrm{w} \%$ & $\mathrm{w} / \mathrm{w} \%$ & $\mathrm{w} / \mathrm{w} \%$ & w/w\% & $\mathrm{w} / \mathrm{w} \%$ & $\mathrm{w} / \mathrm{w} \%$ & $\mathrm{w} / \mathrm{w} \%$ \\
\hline Cilostazol & 33.33 & 33.33 & 33.33 & 33.33 & 33.33 & 33.33 & 33.33 & 33.33 \\
\hline Lactose Monohydrate & 16.67 & 16.67 & 16.67 & 16.67 & 16.67 & 16.67 & 16.67 & 16.67 \\
\hline Polyethylene Glycol 4000 & - & 5.0 & 10.0 & 20.0 & - & 5.0 & 10.0 & 20.0 \\
\hline Purified Water* & - & - & - & - & q. s $^{* *}$ & $\mathrm{q} \cdot \mathrm{s}^{* *}$ & q. s $^{* *}$ & q.s s* $^{* *}$ \\
\hline Avicel PH102 & 46.0 & 41.0 & 36.0 & 26.0 & 46.0 & 41.0 & 36.0 & 26.0 \\
\hline Carmellose Calcium & 2.0 & 2.0 & 2.0 & 2.0 & 2.0 & 2.0 & 2.0 & 2.0 \\
\hline Colloidal Silicon Dioxide & 1.0 & 1.0 & 1.0 & 1.0 & 1.0 & 1.0 & 1.0 & 1.0 \\
\hline Mg Stearate & 1.0 & 1.0 & 1.0 & 1.0 & 1.0 & 1.0 & 1.0 & 1.0 \\
\hline Total & 100.0 & 100.0 & 100.0 & 100.0 & 100.0 & 100.0 & 100.0 & 100.0 \\
\hline
\end{tabular}

* Evaporated during the drying process, though it is not included in the final tablets weight. ${ }^{* *}$ Sufficient quantity.

as described before using a rotary compression machine equipped with the same tooling.

\section{Hot-melt extrusion process}

A dry physical blend of Cilostazol, PEG4000 polymer, and Lactose Monohydrate for each formulation was fed into the hopper of a vertical lab-scale single screw Microtruder (Model RCP-0375) with a screw diameter of 0.3750 inches and a working length to diameter ratio of 24 (Randcastle Extrusion Systems Inc., Cedar Grove, NJ, USA). A rod-shaped die was attached to the end of the barrel with a diameter of $4.0 \mathrm{~mm}$. The trails were processed at a screw speed of $20 \mathrm{rpm}$ and at $50,60,65$, and $70{ }^{\circ} \mathrm{C}$ for zone 1,2 , 3 , and the die, respectively. After cooling down to room temperature, the obtained extrudates were manually cut into small parts prior to milling by Sanyo grinder. The milled powder was passed through 30 \# mesh sieve. The addition of extra-granular components and tableting followed the previously described procedures.

Bulk and tapped densities, Hausner Ratio, Carr's index and angle of repose of powder blends

The bulk and tapped densities were measured for the final blends after processing by different formulation techniques. The bulk density was calculated by measuring the weight of a defined volume of each blend based on the following equation

$$
\text { Bulk density }=\frac{\text { Weight }(\mathrm{gm})}{\text { Volume }(\mathrm{ml})}
$$

The tapped density was obtained by mechanically tapping a graduated cylinder containing the sample until little further volume change was observed. The tapped density was measured for the final tapped volume $\left(V_{f}\right)$ for a defined weight of blend as per the following equation:

$$
\text { Tapped density }=\frac{\text { Weight }(\mathrm{gm})}{\mathrm{V}_{\mathrm{f}}(\mathrm{ml})}
$$

The compressibility index or Carr's index (CI) and the Hausner ratio are calculated from the unsettled apparent volume $\mathrm{V}_{0}$ and the final tapped volume $\mathrm{V}_{\mathrm{f}}$ after no further volume changes occur according to the following equations:

$$
\mathrm{CI}=\frac{\mathrm{V}_{0}-\mathrm{V}_{\mathrm{f}}}{\mathrm{V}_{0}} \times 100 \%
$$

$$
\text { Hausner ratio }=\frac{\mathrm{V}_{0}}{\mathrm{~V}_{\mathrm{f}}}
$$

Finally, the angle of repose was calculated by measuring the angle of the free surface of a pile of powder to the horizontal plane according to the following equation:

$$
\operatorname{Tan}(\alpha)=\frac{\text { Height }}{0.5 \text { base }}
$$

During all experiments, the height of the funnel was fixed at $2.5 \mathrm{~cm}$. The diameters of the base formed were measured carefully. The powder of each formula passed through the funnel without any intervention. All of the cones formed were symmetrical.

\section{Particle size distribution}

Particle size distribution of different formulations was tested by laser diffraction technology. Enough quantity of the blend of each formulation was placed in the dry sampling part (Aero) of a Mastersizer 3000 (Malvern, model 3000, Worcestershire-UK). The powder was passed through the dry cell with a laser beam focused through a glass window inside the cell. Based on the diffracted laser beams, the instrument software provided the cumulated particle size distribution. $\mathrm{D}_{10}, \mathrm{D}_{50}$, and $\mathrm{D}_{90}$ values were calculated for each formulation, where they represent $10 \%$, $50 \%$, and $90 \%$ volume of the particle mass diameter that is smaller than the size indicated, respectively.

\section{Physical characterization of the tablets: weight, hardness, thickness and disinte- gration testing}

Target hardness was set to be around 100 Newton for all the trials in order to exclude any possible effect on the dissolution profile except for factors were set by the study; i.e., polymer quantity and the method of formulation. Samples from the prepared formulations were randomly selected and tested for weight variation, crushing strength and thickness. The weight variation test was conducted by 
weighing 10 tablets from each formulation individually using a Mettler Toledo electronic lab balance. The individual tablet weights were compared to the average. The acceptance criterion for the weight variation is $5 \%$ from average weight (general chapter, USP40). The hardness and thickness of 4 tablets from each formulation were determined using a calibrated hardness tester; ERWEKA TBH300MD hardness tester (Langen, Germany). The force in Newton needed to break each tablet was recorded side by side to the thickness of each sample. The mean, SD and RSD were calculated. Disintegration of 6 tablets of each trial was measured in a warmed tap water $37^{\circ} \mathrm{C}$ temperature, using Electrolab Disintegration Tester (Electrolab, model ED-L2, India). The results from different characterization procedures were recorded and the mean value, SD and RSD were calculated for each trial.

\section{In-vitro dissolution}

The dissolution was performed in a paddle apparatus with a stirring rate of $75 \mathrm{rpm}$ in a medium consisted of $900 \mathrm{ml}$ with $0.3 \%$ Sodium Lauryl Sulfate solution maintained at $37 \pm 0.5^{\circ} \mathrm{C}$ (USP 40). A Pharmatest dissolution system (Model PTW II; Hamburg, Germany) was used. Five milliliters of the test fluid were withdrawn manually at predetermined time interval $(5,10,15,20,30,45$, and $60 \mathrm{minu}$ tes), passed through a $0.45-\mu \mathrm{m}$ milli-pore glass filter and analyzed for Cilostazol at $\lambda=259 \mathrm{~nm}$ by means of a UVVIS single-beam spectrophotometer (Model 9200; United Kingdom). At each sampling time, a pre-warmed equal volume of the test medium was replaced. All the measurements were performed in duplicates and the amount of Cilostazol released for 6 tablets per each formula was determined using a respective calibration curve.

\section{Differential scanning calorimetry}

DSC technique was used in order to recognize any changes that could happen to the API crystal form after incorporating into HME system with the hydrophilic polymer. The DSC scans of the API, each polymer, and the main filler were performed separately. Further combination of the API with the main filler, with and without PEG4000 were tested by the DSC as well. Finally, DSC scans of the different extrudates were performed. A sample with a range of 5 to $10 \mathrm{mg}$ of each trial was weighed inside the crucible, and then the crucible was sealed. The sample was inserted next to a reference crucible inside the DSC furnace maintained with $\mathrm{N}_{2}$ inert gas flow of $100 \mathrm{~cm} /$ minute. The samples were scanned at a heat rate of $10^{\circ} \mathrm{C} /$ minute from ambient temperature to $200^{\circ} \mathrm{C}$, except for lactose monohydrate sample which was scanned to $250^{\circ} \mathrm{C}$.

\section{Results and discussion}

Tablets dosage form is the most convenient route of administration. In contrast, it has a main drawback regar- ding poor solubility for many APIs in the pharmaceutical pipeline. Hydrophilic polymers are expected to enhance the dissolution profile of poorly soluble drugs (Xu et al. 2007; Patel and Patel 2008). However, the magnitude of enhancement is dependent on the type and quantity of the hydrophilic polymer used as well as the principle method of formulation or manufacturing(Hashim et al. 2015; Patel et al. 2008).Understanding excipient functionality is a prerequisite for a successful product formulation development (Aljaberi et al. 2009; Aljaberi et al. 2013a, b). Therefore, a poorly soluble API, Cilostazol, with very bad physical characteristics was selected for the purpose of this study. Various trials using DC, WG, and HME with the incorporation of PEG4000 at various levels were under analysis to illustrate the impact of manufacturing technique on the excipient functionality of this hydrophilic polymer.

\section{Physical characteristics of the final blends}

The physical characteristics of each of the DC, WG, HME trials were measured as detailed in the experimental part and the results are summarized in Table 2.The results show that DC method had low contribution with the physical characteristics of the blends i.e. the API implied its bad nature over the blends of DC trials. Bad flow and sticky nature of the blend were the general characteristics of all the DC trials. No real enhancement for the flow upon incorporation of the polymers could be observed; even at the highest concentrations. The same could be seen with the WG method when PEG4000 was not incorporated. WG01 was with the worst characteristics in WG trials. However, it was better than all the DC trials. The incorporation of the polymer to the formulation had a positive effect on the flow of the powder. In agreement with the previously reported improvement of flowability and compactibility, HME implied the best physical characteristics over all other trials (Pinho et al. 2018). Melting the polymeric material with vigorous mixing and compression, followed by milling resulted in a powder with better characteristics. HME with PEG4000 was with slightly better results when compared to WG with PEG4000 at the same level. The more the quantity of PEG4000 used, the less the difference between WG and HME in terms of physical characteristics of the final blends. It appears also that the more the quantity of the polymer used, the better the characteristics of the final blend (Table 2)

\section{Particle size distribution of the final blends}

The results of particle size distribution of each of the DC, WG and HME trials are shown in Table 3. Since no particle size modification was employed in the DC process, two main peaks could be observed from the PSD analysis. The first for the API within the range of 10 microns and the other peak was for the rest of the composition around 200 
Table 2. Physical characteristics for the Cilostazol Final blends.

\begin{tabular}{|c|c|c|c|c|c|c|c|c|c|c|}
\hline Trial number & DC01 & DC02 & DC03 & DC04 & WG01 & WG02 & WG03 & WG04 & HME02 & HME03 \\
\hline $\begin{array}{l}\text { Apparent Density } \\
(\mathrm{gm} / \mathrm{ml})\end{array}$ & 0.31 & 0.32 & 0.33 & 0.34 & 0.46 & 0.46 & 0.51 & 0.51 & 0.52 & 0.60 \\
\hline $\begin{array}{l}\text { Tapped density } \\
(\mathrm{gm} / \mathrm{ml})\end{array}$ & 0.58 & 0.56 & 0.56 & 0.55 & 0.73 & 0.63 & 0.66 & 0.63 & 0.65 & 0.74 \\
\hline Carrs' index & 85.19 & 72.41 & 70.12 & 61.29 & 60.00 & 36.05 & 29.03 & 23.46 & 25.00 & 22.70 \\
\hline Hausner ratio & 1.85 & 1.72 & 1.70 & 1.61 & 1.60 & 1.36 & 1.29 & 1.23 & 1.25 & 1.23 \\
\hline Angle of repose & 52.8 & 51.0 & 49.6 & 47.2 & 42.6 & 36.4 & 32.3 & 30.1 & 29.4 & 27.8 \\
\hline Flow according to $\mathrm{CI}$ & Very very poor & Very very poor & Very very poor & Very very poor & Very very poor & Very poor & Poor & Passable & Passable & Passable \\
\hline $\begin{array}{l}\text { Flow according to } \\
\text { Hausner ratio }\end{array}$ & Very very poor & Very very poor & Very very poor & Very very poor & Very poor & Poor & Passable & Fair & Fair & Fair \\
\hline $\begin{array}{l}\text { Flow according to } \\
\text { Angle of repose }\end{array}$ & $\begin{array}{c}\text { Poor - must } \\
\text { agitate or vibrate }\end{array}$ & $\begin{array}{c}\text { Poor - must } \\
\text { agitate or vibrate }\end{array}$ & $\begin{array}{c}\text { Poor - must } \\
\text { agitate or vibrate }\end{array}$ & $\begin{array}{c}\text { Poor - must } \\
\text { agitate or vibrate }\end{array}$ & Passable & Fair & Good & Excellent & Excellent & Excellent \\
\hline
\end{tabular}

Table 3. Particle size distribution of Cilostazol final blends.

\begin{tabular}{lccc}
\hline \multicolumn{1}{c}{ Trial number } & $\mathbf{D}_{\mathbf{1 0}}(\mu \mathrm{m})$ & $\mathbf{D}_{\mathbf{5 0}}(\mu \mathrm{m})$ & $\mathbf{D}_{\mathbf{9 0}}(\mu \mathrm{m})$ \\
\hline DC01 & 3.18 & 46.8 & 256.0 \\
DC04 & 3.29 & 46.3 & 261.0 \\
WG01 & 6.42 & 90.2 & 316.0 \\
WG02 & 9.21 & 100.0 & 329.0 \\
WG03 & 13.7 & 123.0 & 362.0 \\
WG04 & 24.3 & 135.0 & 379.0 \\
HME02 & 20.1 & 132.0 & 374.0 \\
HME03 & 27.3 & 145.0 & 386.0 \\
\hline
\end{tabular}

microns. No significant changes on the PSD were noticed after adding PEG4000. These results were endorsed by the bad physical characteristics of the blends for all the DC trials. On the other hand, the WG and HME trials resulted in a more homogeneous particle sizes distribution. As the quantity of the polymer used was increased, so did the size and homogeneity of the final blend.

\section{Physical characteristics of the tablets}

The physical characteristics for the compressed tablets of each of DC, WG and HME formulations were recorded and they are summarized in the Table 4 . Tablets produced with DC method had sticking on the surface. There was a weight variation problem during the compression process. Tablets with weight that exceeded the allowable limits were excluded. The applied force for the compression was adjusted to get final tablets with hardness value around $100 \mathrm{~N}$. This hardness value was granted for all the trials in order to avoid any effect of the tablets hardness upon the dissolution profile. Disintegration time of DC tablets was very fast and did not exceed one minute in most of the trials.

Table 4. Physical characteristics of the Cilostazol tablets.

\begin{tabular}{lccc}
\hline & $\begin{array}{c}\text { Tablets weight }(\mathbf{m g}) \\
\mathbf{n}=\mathbf{1 0}\end{array}$ & $\begin{array}{c}\text { Tablet hardness (N) } \\
\mathbf{n}=\mathbf{4}\end{array}$ & $\begin{array}{c}\text { Disintegration (min) } \\
\mathbf{n}=\mathbf{6}\end{array}$ \\
\hline DC01 & $301.6 \pm 3.5$ & $99.3 \pm 8.6$ & 0.50 \\
DC02 & $299.6 \pm 3.5$ & $103.0 \pm 5$ & 0.24 \\
DC03 & $302.9 \pm 3.5$ & $99.9 \pm 4$ & 0.50 \\
DC04 & $303.7 \pm 6.1$ & $101.8 \pm 7$ & 1.01 \\
WG01 & $300.3 \pm 1.4$ & $102.8 \pm 4.3$ & 0.34 \\
WG02 & $301.2 \pm 1.7$ & $109.0 \pm 4.1$ & 3.45 \\
WG03 & $301.3 \pm 1.9$ & $105.3 \pm 3.9$ & 8.05 \\
WG04 & $300.4 \pm 1.3$ & $103.3 \pm 3.8$ & 35.17 \\
HME02 & $300.4 \pm 1.0$ & $104.8 \pm 2.6$ & 11.00 \\
HME03 & $300.9 \pm 1.9$ & $105.8 \pm 3.3$ & 37.83 \\
\hline
\end{tabular}

Compressed tablets using WG method without PEG4000 (WG01) suffered sticking issues, but the tablets weight was stable at the minimum speed of the compression machine. These tablets resulted in a fast disintegration time $(\sim 30$ seconds). When PEG was incorporated, tablets produced in trials WG02, WG03 and WG04 were with good shining appearance and no sticking problem on the punches. Disintegration time varied depending on the quantity of the polymer used, and it was increasing exponentially with increasing the amount of the polymer (Table 4).

Tablets produced by the HME technique were also with a good shining appearance and no sticking problems. Tablets weight during the compression was stable even at the maximum speed of the compression machine. Disintegration behavior was similar to that of the WG tablets. Comparison of the disintegration time for tablets with the same composition produced by the three manufacturing techniques shows that HME tablets were always with the longest disintegration time.

Overall, when using PEG4000, tablets produced by HME had less thickness values compared to tablets with the same composition prepared by WG. Disintegration time was slightly higher for HME tablets when compared to similar formulations prepared by WG. As per the disintegration time, it increased almost four times at $20 \%$ PEG4000 trials when compared to 10\% PEG4000 trials for both HME and WG trials.

\section{In-vitro dissolution results}

The most obvious impact of the manufacturing technique was on the dissolution of prepared tablets. The dissolution profiles for different trials were tested according to the dissolution test stated in the United State Pharmacopeia for Cilostazol tablets. As can be noticed in Fig. 2, different DC trials showed a fast release. However, it was noticed that none of the DC trials reached a full release profile at the end of the dissolution run time. This reflects that the incorporation of a hydrophilic polymer in the DC technique had a low contribution to the dissolution of Cilostazol. F2 values between the standard formulation DC01 and the other DC trails were $>50$; indicating that there are no significant differences even at the maximum level of PEG4000. Although the dissolution of DC tablets was satisfactory, the tablets prepared by this method suffered hampering 


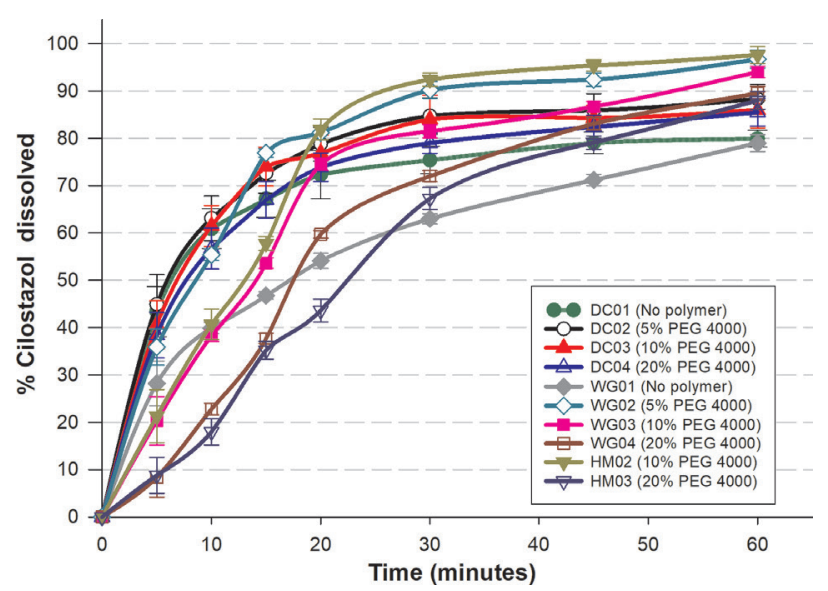

Figure 2. Dissolution profiles of Cilostazol tablets prepared using various manufacturing techniques (Error bars represent the standard deviation, $\mathrm{n}=6$ ).

weight variation and stickiness issues which rendered the technique unsuitable for further consideration.

While being nonfunctional in the DC trials, the PEG4000 level had a significant influence on the release of the model drug from WG and HME tablets. Moreover, the tablets experienced different behaviors at different PEG4000 ratios with different manufacturing techniques. This behavior varied from enhancement to retardation of Cilostazol dissolution as compared to the DC trails. Interestingly, the WG01 formulation with no PEG4000 showed a slow dissolution which did not exceed $80.0 \%$ of Cilostazol. The addition of $5.0 \%$ of PEG4000 enhanced the dissolution significantly with an average release of $96.7 \%$ at the end of the release. At this level, the release was as fast as those of the DC trials but with at least $10 \%$ more release at 60 minutes. Expectedly, the significant enhancement of the dissolution of Cilostazol was confirmed by an $\mathrm{f} 2$ value of 31.8 between WG01 and WG02 tablets. When the level of PEG4000 was increased to $10 \%$ (WG03), a retardation of the release rate could be seen as compared to WG02 but with an average release of $94 \%$ at 60 minutes. It is anticipated that the slow disintegration time in WG03 had contributed in the slow liberation of the API. Nevertheless, the $\mathrm{f} 2$ value for WG03 versus WG01 was 43.3 indicating that it is still better than not to incorporate any polymer at all. The more polymer quantity used in WG04 (20\% w/w PEG4000) resulted in a slower disintegration time and consequently in a slower release profile. At the end of the dissolution testing for WG04, it could be seen that the vessels contained small fragments of the tablets that were undissolved.

The dissolution profiles for different HME trials were also investigated. No standard formulation without PEG4000 or at $5 \%$ level can be obtained as the process of HME implies to insert a polymeric molten material in enough quantities. Several attempts to extrude the $5 \%$ PEG4000 formulation were investigated with some modifications to the temperatures of the different zones of the extruder and on the rotation speed of the screw. Nevertheless, the blend was with poor extrudability due to the inconvenient flow of the molten polymer with the API: Lactose Monohydrate mixture inside the barrel. HME02 extrudates was extruded slowly with a clay-appearance, while HME03 was extruded smoothly as per the processing parameters specified in the methods section.

For trials with PEG4000 at 10\%, HME02 had exceeded the other trials and had given a $90 \%$ release at 30 minutes in spite of the slow start. This slow start can be explained by the slow disintegration of tablets in HME02 and to a less extent in WG03. Once HME02 tablets disintegrated, they eluted Cilostazol quickly compared to WG03 and DC03. At the end of the dissolution runs, both HME02 and WG03 had almost a full release of the API while it was only $86 \%$ for DC03. This can be understood as the homogeneity of the polymer distribution and spreading was better by the HME technique followed by WG and worst for the DC technique.

On the other hand, adding 20\% of PEG4000 slowed down the dissolution in trial HME03 without a full release of the API at 60 minutes. Tablets in dissolution vessels were observed with an almost unchanged shape for the first 30 minutes of the dissolution run. HME03 tablets disintegrated very slowly; they needed 38 minutes to be fully disintegrated in the disintegration tester (Table 4). It is believed that $20 \%$ of PEG 4000 had formed a hydrophilic matrix in tablets of trial HME03 and to lower extent in WG04, thus Cilostazol eluted slower in the trial HME03 than that of WG04. This can be understood as HME distributed PEG4000 more efficiently than WG and therefore, the hydrophilic matrix that sustained the release in HME03 was more efficient in that aspect than it in WG04. When compared to the trials with 10\% PEG4000, HME and WG with 20\% PEG4000 had a slower release profile. Although HME03 had a slower release compared to WG04, f2 value revealed some kind of similarity between the dissolution profiles of HME03 and WG04 (f2 value 54.1).

\section{Differential scanning calorimetry}

Among other techniques, DSC is beneficial in the characterization of the solid state of the drug in extrudates (Censi et al. 2018). To set a reference point, DSC testing for Cilostazol, Lactose Monohydrate and a combination of Cilostazol: Lactose Monohydrate (2:1) were done separately in order to standardize any possible changes after the different manufacturing processes. Cilostazol showed a main endothermic peak at $159^{\circ} \mathrm{C}$, while Lactose Monohydrate showed two main endothermic peaks; one of them represents the dehydration of Lactose at $145^{\circ} \mathrm{C}$ and the other is at $218^{\circ} \mathrm{C}$ and represents the melting of Lactose (Raut et al. 2011). The melting of Lactose was beyond the studied range for other trials as the important peaks were for the API and PEG4000, where all of them were below $200{ }^{\circ} \mathrm{C}$. The mixture of Cilostazol and Lactose Monohydrate showed two main peaks without any changes (Figure 3). In addition, DSC testing was done to PEG4000. A main endothermic peak was observed at $64^{\circ} \mathrm{C}$ for PEG4000 as seen in Fig. 3. 


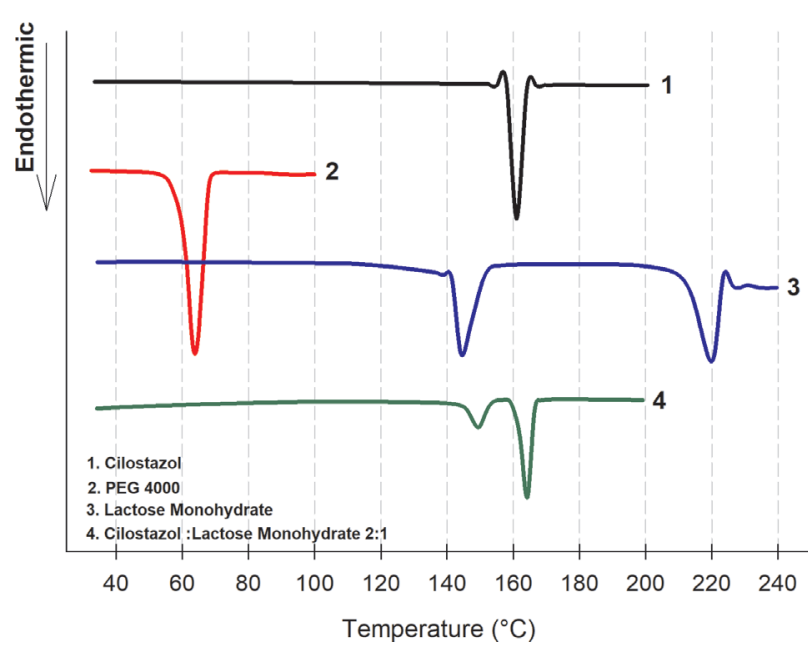

Figure 3. DSC scans of raw materials as well as Cilostazol: Lactose Monohydrate at the ratio used in preparing the tablets by various manufacturing techniques (Table 1).

DSC testing for the trials DC04, WG04 and HME03 was undertaken as these trials contain the maximum amount of PEG4000. The DSC patterns showed no significant changes on any of the materials regarding the place or the intensity/weight ratios of the PEG4000 and Cilostazol peaks as observed in Figure 4. A gradual change in the onset of Lactose dehydration peak was noticed, in a matter that is dependent on the different formulation processes. The peak of Lactose Monohydrate dehydration appeared to be broader and shallower at HME03 when compared to WG04 and DC04 which may be attributed to partial dehydration of lactose monohydrate during the HME process.

In summary, this work has clarified how the different manufacturing technologies had contributed to Cilostazol dissolution without changing the crystalline form of the API. Unlike the DC trials which were found with no significant differences, WG and HME showed contrasting enhancement and retardation effects regarding the dissolution profile of Cilostazol tablets in a matter that is dependent on the type and quantity of PEG4000 polymer used. Inserting 5\% of PEG4000 in WG had resulted in tablets with a fast dissolution profile. HME with $10 \%$ polymer had resulted in a faster dissolution profile

\section{References}

Albers J, Alles R, Matthée K, Knop K, Nahrup JS, Kleinebudde P (2009) Mechanism of drug release from polymethacrylate-based extrudates and milled strands prepared by hot-melt extrusion. European Journal of Pharmaceutics and Biopharmaceutics 71(2): 387-394. https:// doi.org/10.1016/j.ejpb.2008.10.002

Aljaberi A, Chatterji A, Shah NH, Sandhu HK (2009) Functional performance of silicified microcrystalline cellulose versus microcrystalline cellulose: a case study. Drug development and industrial pharmacy 35(9): 1066-1071. https://doi.org/10.1080/03639040902774131

Aljaberi A, Chatterji A, Dong Z, Shah NH, Malick W, Singhal D, Sandhu HK (2013a) Understanding and optimizing the dual excipient functionality of sodium lauryl sulfate in tablet formulation of poorly

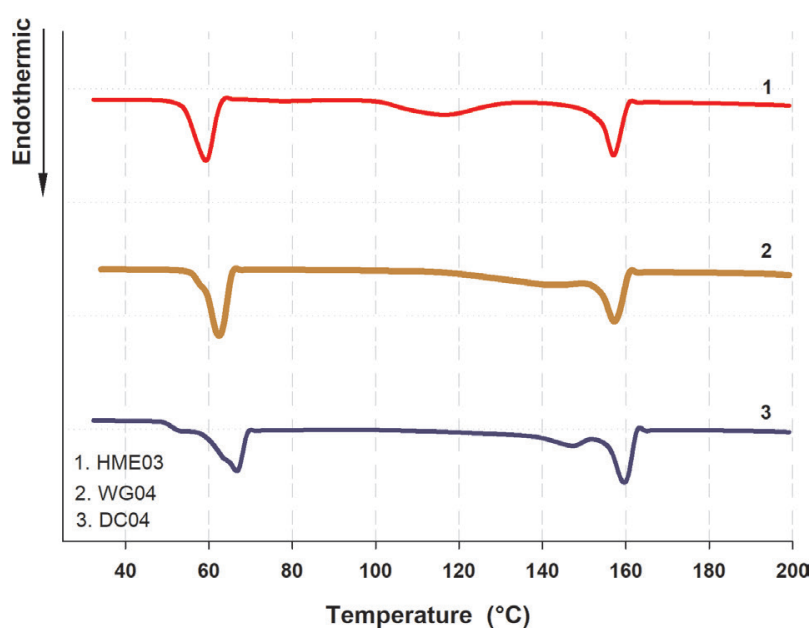

Figure 4. DSC scans of Cilostazol: Lactose Monohydrate: PEG4000 (1) extrudates, (2) WG intragranular components and (3) DC physical mixture, at 20\% level of PEG4000.

compared to WG trials with similar quantities of polymers. The suggested interpretation for the dissolution enhancement at certain concentrations of PEG4000 was referred to a better distribution of the polymer throughout the blends; consequently, better wetting and solubilization. The more quantity of PEG4000 polymer inserted in WG and HME, the slower the dissolution profile was. Long disintegration times for trials with $20 \% \mathrm{w} / \mathrm{w}$ of PEG4000 endorsed this observation. These effects are not related to changes in the solid-state changes of Cilostazol as confirmed by DSC. Overall, the use of the hydrophilic polymer at optimum concentrations was useful as a wetting agent for developing formulations with better dissolution profile.

\section{Acknowledgements}

All the materials were kindly donated by Tabuk Pharmaceutical Research Company (Amman, Jordan).

Authors are grateful to the Applied Science Private University, Amman, Jordan for the full financial support granted to this research project. water soluble drug: wetting and lubrication. Pharmaceutical development and technology 18(2): 490-503. https://doi.org/10.3109/10 837450.2012.723717

Aljaberi A, Ardakani A, Khdair A, Abdel-Rahim SA, Meqdadi E, Ayyash M, Alobaidi GM, Al-Zoubi N (2013b) Tableting functionality evaluation of Prosolv Easytab in comparison to physical mixtures of its individual components. Journal of Drug Delivery Science and Technology 23: 499-504. https://doi.org/10.1016/S1773-2247(13)50072-4 Censi R, Gigliobianco MR, Casadidio C, Di Martino P (2018) Hot melt extrusion: Highlighting physicochemical factors to be investigated while designing and optimizing a hot melt extrusion process. Pharmaceutics 10(3): e89. https://doi.org/10.3390/pharmaceutics10030089 
Hashim II, Ghazy NF, El-Shabouri MH (2015) Potential use of phospholipids in combination with hydrophilic carriers for enhancement of the dissolution and oral bioavailability of imidazole antifungal class II drugs. Die Pharmazie - An International Journal of Pharmaceutical Sciences 70(11): 706-715. https://doi.org/10.1691/ph.2015.5079

Kalepu S, Nekkanti V (2015) Insoluble drug delivery strategies: review of recent advances and business prospects. Acta Pharmaceutica Sinica B 5(5): 442-453. https://doi.org/10.1016/j.apsb.2015.07.003

Kalivoda A, Fischbach M, Kleinebudde P (2012) Application of mixtures of polymeric carriers for dissolution enhancement of oxeglitazar using hot-melt extrusion. International journal of pharmaceutics 439(1-2): 145-156. https://doi.org/10.1016/j.ijpharm.2012.10.013

Leuner C, Dressman J (2000) Improving drug solubility for oral delivery using solid dispersions. European journal of Pharmaceutics and Biopharmaceutics 50(1): 47-60. https://doi.org/10.1016/S09396411(00)00076-X

Maniruzzaman M, Boateng JS, Snowden MJ, Douroumis D (2012) A review of hot-melt extrusion: process technology to pharmaceutical products. International Scholarly Research Notices 2012: 436763. https://doi.org/10.5402/2012/436763

Patel R, Patel M (2008) Preparation, characterization, and dissolution behavior of a solid dispersion of simvastatin with polyethylene glycol 4000 and polyvinylpyrrolidone K30. Journal of dispersion science and technology 29(2): 193-204. https://doi. org/10.1080/01932690701706946

Patel RP, Patel DJ, Bhimani DB, Patel JK (2008) Physicochemical characterization and dissolution study of solid dispersions of furosemide with polyethylene glycol 6000 and polyvinylpyrrolidone K30. Dissolution Technologies 15(3): 17-25. https://doi.org/10.14227/ DT150308P17

Patil H, Tiwari RV, Repka MA (2016) Hot-melt extrusion: from theory to application in pharmaceutical formulation. AAPS Pharmscitech 17(1): 20-42. https://doi.org/10.1208/s12249-015-0360-7

Pinho LA, Souza SG, Marreto RN, Sa-Barreto LL, Gratieri T, Gelfuso GM, Cunha-Filho M (2018) Dissolution enhancement in cocoa extract, combining hydrophilic polymers through hot-melt extrusion. Pharmaceutics 10(3): e135. https://doi.org/10.3390/pharmaceutics 10030135

Raut DM, Allada R, Pavan KV, Deshpande G, Patil D, Patil A, Deshmukh A, Raut DM, Sakharkar DM, Bodke PS, Mahajan DT (2011) Dehydration of lactose monohydrate: analytical and physical characterization. Der Pharmacia Lettre 3(5): 202-212. https://www.researchgate.net/ profile/Dr_Pavan_Kommavarapu/publication/265376074_Dehy-
dration_of_Lactose_Monohydrate_Analytical_and_Physical_Characterization/links/5409dfa50cf2f2b29a2cc7c5/Dehydration-of-Lactose-Monohydrate-Analytical-and-Physical-Characterization.pdf

Regev G, Patel SK, Moncla BJ, Twist J, Devlin B, Rohan LC (2019) Novel Application of Hot Melt Extrusion for the Manufacturing of Vaginal Films Containing Microbicide Candidate Dapivirine. AAPS PharmSciTech 20(6): e239. https://doi.org/10.1208/s12249-019-1442-8

Ren Y, Mei L, Zhou L, Guo G (2019) Recent perspectives in hot melt extrusion-based polymeric formulations for drug delivery: applications and innovations. AAPS PharmSciTech 20(3): e92. https://doi. org/10.1208/s12249-019-1300-8

Repka MA, Majumdar S, Kumar Battu S, Srirangam R, Upadhye SB (2008) Applications of hot-melt extrusion for drug delivery. Expert opinion on drug delivery 5(12): 1357-1376. https://doi. org/10.1517/17425240802583421

Sanoufi MR, Aljaberi A, Hamdan I, Al-Zoubi N (2020) The use of design of experiments to develop hot melt extrudates for extended release of diclofenac sodium. Pharmaceutical Development and Technology 25(2): 187-196. https://doi.org/10.1080/10837450.2019.1684519

Tang B, Liu Z, Tian Z, Zhang J, Chen X, Fang G, Song H (2019) Development and evaluation of synchronized and sustained release Tripergium Wilfordii tablets based hot-melt extrusion and direct powder compression. Journal of Drug Delivery Science and Technology 53: e101208. https://doi.org/10.1016/j.jddst.2019.101208

Weatherley S, Mu B, Thompson MR, Sheskey PJ, O'Donnell KP (2013) Hot-melt granulation in a twin screw extruder: effects of processing on formulations with caffeine and ibuprofen. Journal of pharmaceutical sciences 102(12): 4330-4336. https://doi.org/10.1002/jps.23739

Xu L, Li SM, Sunada H, Wang QF (2007) Improvement of dissolution rate of arbidol hydrochloride from solid dispersion prepared with PEG system by fusion method. Journal of drug delivery science and technology 17(2): 145-148. https://doi.org/10.1016/S17732247(07)50022-5

Yang Z, Hu Y, Tang G, Dong M, Liu Q, Lin X (2019) Development of ibuprofen dry suspensions by hot melt extrusion: Characterization, physical stability and pharmacokinetic studies. Journal of Drug Delivery Science and Technology 54: e101313. https://doi.org/10.1016/j. jddst.2019.101313

Zi P, Zhang C, Ju C, Su Z, Bao Y, Gao J, Sun J, Lu J, Zhang C (2019) Solubility and bioavailability enhancement study of lopinavir solid dispersion matrixed with a polymeric surfactant-Soluplus. European Journal of Pharmaceutical Sciences 134: 233-245. https://doi. org/10.1016/j.ejps.2019.04.022 